\title{
ОТДАЛЕННЫЕ РЕЗУЛЬТАТЫ ПРЕВЕНТИВНОГО БЛОКА КАВАТРИКУСПИДАЛЬНОГО ПЕРЕШЕЙКА В ИНТЕРВЕНЦИОННОМ ЛЕЧЕНИИ ФИБРИЛЛЯЦИИ ПРЕДСЕРДИЙ
}

\section{LONG-TERM RESULTS OF THE PREVENTIVE BLOCK OF THE CAVATRICUSPIDAL ITSTHUS IN INTERVENTIONAL TREATMENT OF ATRIAL FIBRILLATION}

N. Safonov

V. Baranovich

A. Faibushevich

Summary. The purpose of this study: To determine the long-term results of preventive block in the cavatricuspid isthmus during isolation of the pulmonary vein orifices.

The study was conducted from 2018 to 2020. Inclusion criteria: paroxysmal $A F$, absence of atrial fibrillation. Presence of risk factors for TP. Exclusion criteria: typical or atypical LT. The study included 42 patients. The first group performed only isolation of the pulmonary vein orifices. The second group is the isolation of the orifices of the pulmonary veins simultaneously with the ablation of the CTP. The primary endpoint is the absence of AF and AT during the blind period. The secondary endpoint was the absence of AF paroxysms documented by ECG or HM-ECG (duration $60 \mathrm{~s}$ ) after the blind period ( 3 months), the occurrence of typical atrial flutter atrial flutter.

Pulmonary vein isolation criteria were obtained in all patients. The efficiency of (TP ablation was $100 \%$. During the blind period (3 months), AF paroxysms were recorded in 5 patients (23.81\%) patients in the first group and in 4 patients (19.05\%) in the second group. The efficiency of the operation within one year (efficiency in atrial fibrillation) in the first group was $(76.19 \%)$, in the second group (71.43\%). No recurrence of typical atrial flutter was observed in the second group. In the first group, there were $3(14.29 \%)$ cases of TP. Below is table No. 4 for the risk factors of recurrent patients (TP) from the first group. Efficiency for atrial fibrillation in the first group within two years was $66.67 \%$ (14 patients), in the second group $61.9 \%$ ( 13 patients). There were no cases of TP in the second group after 2 years of follow-up. In the first group, 4 cases of TP were identified within two years (19.05\%).

It is possible to perform preventive ablation in patients with risk factors for AT, especially in patients with multiple risk factors for AT. Preventive ablation of CTP insignificantly increases the operation time and does not increase the number of intraoperative complications. Preventive ablation of CTP insignificantly lengthens the operation time, does not affect the occurrence of complications, and can reduce the number of subsequent hospitalizations and reoperations.
Сафонов Никита Владимирович

Aспирант, ФГАОУ ВО «Российский университет дружбы народов» safon_92@mail.ru

Баранович Владислав Юрьевич К.м.н., дочент, ФГАОУ ВО «Российский университет дружбы народов» vubara@yandex.ru

Файбушевич Александр Георгиевич К.м.н., ФГАОУ ВО «Российский университет дружбы народов»

faybushevich_ag@pfur.ru

Аннотация. Цель данного исследования: Определить отдаленные результаты превентивного блока в каватрикуспидальном перешейке при проведении изоляции устьев легочных вен.

Исследование проводилось с 2018 по 2020 годы. Критерии включения: пароксизмальная ФП, отсутствие ТП. Наличие факторов риска ТП. Критерии исключения: типичное или атипичное ТП. В исследование включено 42 пациента. Первая группа проводилась только изоляция устьев лёгочных вен. Вторая группа изоляция устьев легочных вен одномоментно с абляцией КТП. Первичная конечная точка - отсутствие ФП и ТП во время слепого периода. Вторичной конечной точкой было отсутствие пароксизмов ФП, задокументированных на ЭКГ или по ХМ-ЭКГ (длительность 60 с) после слепого периода (3 мес), возникновение типичного трепетания предсердий ТП.

Критерии изоляции легочных вен были получены у всех пациентов. Эффективность абляции КТП составила 100\%. В течение слепого периода (3 месяца) пароксизмы ФП регистрировались у 5 пациентов $(23,81 \%)$ пациентов в первой группе и у 4 пациентов (19,05\%) во второй группе. Эффективность операции в течение одного года (эффективность по фибрилляции предсердий) в первой группе составила (76,19\%), во второй группе (71,43\%). Рецидивов типичного трепетания предсердий во второй группе не наблюдалось. В первой группе наблюдалось 3 (14,29\%) случая ТП. Далее представлена таблица № 4 по факторам риска рецидивных пациентов (ТП) из первой группы. Эффективность по фибрилляции предсердий в первой группе в течение двух лет составила $66,67 \%$ (14 пациентов), во второй группе 61,9\% (13 пациентов). Случаев ТП во второй группе через 2 года наблюдения не было. В первой группе в течение двух лет было определено 4 случая ТП (19,05\%).

Возможно проведение превентивной абляции пациентам с факторами риска ТП, особенно пациентам с наличием нескольких факторов риска ТП. Превентивная абляция КТП незначительно увеличивает время операции и не увеличивает число интраоперационных осложнений. Превентивная абляция КТП незначительно удлиняет время операции, не влияет на воз- 
Keywords: atrial fibrillation, atrial flutter, risk factors, radiofrequency ablation. никновение осложнений и способна снизить число последующих госпитализаций и повторных операций.

Ключевые слова: фибрилляция предсердий, трепетание предсердий, факторы риска, радиочастотная абляция.
$\Phi$ ибрилляция предсердий (ФП) — это вид наджелудочковой тахиаритмии с хаотической электрической активностью предсердий с частотой импульсов от 350 до 700 в минуту. Частота встречаемости фибрилляции предсердий в популяции составляет 1,5-2\% [1]. Триггерная активность устьев легочных вен приводит к возникновению и поддержанию фибрилляции предсердий. Интервенционное лечение ФП заключается в изоляции устьев легочных вен, создании так называемого «блока входа» и «блока выхода» [2]. В настоящее время отдаленные результаты изоляции устьев легочных вен составляют около 70\% [3].

Типичное трепетание предсердий (ТП) - вид наджелудочковой аритмии с электрической активностью предсердий, при которой частота импульсов достигает 250-350 ударов в минуту [4]. Электрофизиологически ТП возникает из-за критического замедления проведения в области каватрикуспидального перешейка (КТП). Интервенционное лечение ТП заключается в создании двунаправленного блока проведения по (КТП). Отдаленные результаты интервенционного лечения ТП достигают 95\% в течение 10 лет [5].

ФП и ТП являются разными аритмиями с различным патогенезом, однако довольно часто они диагностируются совместно [5].

В настоящее время согласно современным рекомендациям, при диагностировании ФП и ТП, показано проведение одномоментной операции - абляции КТП и изоляции устьев легочных вен (Класс Ila, уровень В). В настоящее время подавляющее большинство хирургов аритмологов (около 95\%) проводят сочетанную операцию [6]. В настоящее время согласно современным рекомендациям при отсутствии ТП в анамнезе возможно проведение абляции КТП (Класс Ilb, уровень В). Таким образом, вопрос превентивной абляции КТП у пациентов с недокументированным ТП остается открытым. Данная проблема послужила причиной проведения нашей работы.

Исследование проводилось в период с 2018 по 2020 годы. Критерии включения: пароксизмальная форма ФП, отсутствие документированного ТП, наличие факторов риска ТП (ХСН, ХОБЛ, удлиненный интервал p-r, инфаркт миокарда в анамнезе, курение). Критерии исключения: типичное или атипичное трепетание предсердий в ана- мнезе. Конечные точки: основные сердечно-сосудистые события, повторные операции, возникновение ТП. В исследование включено 42 пациента. Пациенты были рандомизированы на 2 группы. Средний возраст 62 4 года. Характеристика пациентов приведена в таблице № 1. Пациенты были включены в исследования после информированного письменного согласия. Исследование одобрено этическим комитетом РУДН. У пациентов из первой группы проводилась только изоляция устьев лёгочных вен. Характеристика пациентов представлена в таблице № 2. У пациентов из второй группы изоляция устьев легочных вен одномоментно с абляцией КТП. Характеристика пациентов представлена в таблице № 3 . Группы пациентов сравнивали с помощью критерия Фишера - различия между группами по частоте встречаемости сопутствующих заболеваний нет $(p<0,05)$. Все пациенты получали антикоагулянтную терапию как минимум за 4 недели до абляции. При терапии варфарином, операция выполнялась при значении МНO 2,1-2,3. Пациенты прекращали прием антиаритмических средств за 2-3 периода полувыведения перед абляцией. Интраоперационно всем пациентам выполнялась трансторакальная и внутрисердечная эхокадиография с целью исключения структурного заболевания сердца и образование тромба в левом предсердии и его ушке. Для проведения внутрисердечного Эхо-КГ использовался аппарат GE Vivid-I, также датчик для внутрисердечного ЭХО-КГ ACUSON AcuNav ${ }^{\mathrm{TM}}$ Ultrasound Catheter.

Первичная конечная точка - отсутствие ФП и ТП во время слепого периода. Вторичной конечной точкой было отсутствие пароксизмов ФП, задокументированных на ЭКГ или по ХМ-ЭКГ (длительность 60 с) после слепого периода (3 мес), возникновение ТП. Расшифровка данных ХМ-ЭКГ проводилась одним и тем же специалистом. Пациенты были снабжены журналом симптомов для записи любых симптомов, похожих на ФП. Записи ЭКГ ХМ-ЭКГ с регулярной предсердной тахикардией были тщательно изучены тремя независимыми электрофизиологами.

\section{Хом операчии}

Трижды пунктировалась правая бедренная вена. Пункция межпредсердной перегородки (МПП) проводилась под контролем внутрисердечного УЗИ. После пункции бедренной вены вводился раствор гепарина (100 мг / кг). 
Таблица 1. Сопутствующие заболеваниям и факторы риска возникновения типичного трепетания предсердий:

\begin{tabular}{|l|l|}
\hline Количество пациентов & $\mathbf{4 2} \mathbf{1 0 0 \%}$ \\
\hline Мужчины & $2047,62 \%$ \\
\hline Женщины & $2252,38 \%$ \\
\hline Сахарный диабет & $511,9 \%$ \\
\hline Хроническая сердечная недостаточность & 36 85,71\% \\
\hline Удлнённый интервал р-г & $2457,14 \%$ \\
\hline Ишемическая болезнь сердца & $1023,81 \%$ \\
\hline Хроническая обструктивная болезнь легких & $1638,1 \% \%$ \\
\hline Артериальная гипертония & $3788,1 \%$ \\
\hline Курение & $1740,48 \%$ \\
\hline Клапанные пороки & 13 30,95\% \\
\hline Хроническая болезнь почек & 4 9,52\% \\
\hline Ожирение & $819,05 \%$ \\
\hline Инфаркт миокарда в анамнезе & $716,67 \%$ \\
\hline
\end{tabular}

Таблица 2. Характеристика пациентов 1 группы

\begin{tabular}{|l|l|}
\hline Количество пациентов & $21 \quad 100 \%$ \\
\hline Мужчины & $942,86 \%$ \\
\hline Женщины & $1257,14 \%$ \\
\hline Сахарный диабет & $314,29 \%$ \\
\hline Хроническая сердечная недостаточность & $1676,19 \%$ \\
\hline Удлинённый интервал р-г & $1361,9 \%$ \\
\hline Ишемическая болезнь сердца & $419,05 \%$ \\
\hline Хроническая обструктивная болезнь легких & $733,33 \%$ \\
\hline Артериальная гипертония & $1980,95 \%$ \\
\hline Курение & $733,33 \%$ \\
\hline Клапанные пороки & $628,57 \%$ \\
\hline Хроническая болезнь почек & 2 9,52\% \\
\hline Ожирение & $314,29 \%$ \\
\hline Инфаркт миокарда в анамнезе & $419,05 \%$ \\
\hline
\end{tabular}

Таблица 3. Характеристика пациентов 2 группы

\begin{tabular}{|l|l|}
\hline Количество пациентов & $\mathbf{2 1} \mathbf{1 0 0 \%}$ \\
\hline Мужчины & $1152,38 \%$ \\
\hline Женщины & $942,86 \%$ \\
\hline Сахарный диабет & 2 9,52\% \\
\hline Хроническая сердечная недостаточность & $2095,24 \%$ \\
\hline Удлинённый интервал р-г & $1152,38 \%$ \\
\hline Ишемическая болезнь сердца & $628,57 \%$ \\
\hline Хроническая обструктивная болезнь легких & $942,86 \%$ \\
\hline Артериальная гипертония & 18 85,71\% \\
\hline Курение & $1047,62 \%$ \\
\hline Клапанные пороки & $628,57 \%$ \\
\hline Хроническая болезнь почек & 2 9,52\% \\
\hline Ожирение & $523,81 \%$ \\
\hline Инфаркт миокарда в анамнезе & $314,29 \%$ \\
\hline
\end{tabular}


Таблица 4. Характеристика рецидивных пациентов из первой группы (ТП)

\begin{tabular}{|c|c|c|c|c|}
\hline Номер пациента & 1 & 2 & 3 & 4 \\
\hline Пол & $M$ & M & * & * \\
\hline Сахарный диабет & + & - & - & Сахарный диабет 2 типа \\
\hline $\begin{array}{l}\text { Хроническая сердечная } \\
\text { недостаточность }\end{array}$ & $\begin{array}{l}\text { Функциональный класс } 2 \\
\text { фракция выброса 47\% }\end{array}$ & $\begin{array}{l}\text { Функциональный класс } 1 \\
\text { фракция выброса 50\% }\end{array}$ & $\begin{array}{l}\text { Функциональный класс } 2 \\
\text { фракция выброса 49\% }\end{array}$ & $\begin{array}{l}\text { Функциональный класс } 1 \\
\text { фракция выброса 53\% }\end{array}$ \\
\hline $\begin{array}{l}\text { Удлинённый интервал } \\
\text { p-r }\end{array}$ & $215 \mathrm{MC}$ & $245 \mathrm{MC}$ & $210 \mathrm{MC}$ & $230 \mathrm{MC}$ \\
\hline $\begin{array}{l}\text { Ишемическая болезнь } \\
\text { сердца }\end{array}$ & $\begin{array}{l}\text { Стенокардия } \\
\text { Функциональный класс } 2\end{array}$ & Нет & $\begin{array}{l}\text { Инфаркт миокарда } 2015 \\
\text { год } \\
\text { Стенокардия } \\
\text { Функциональный класс } 2\end{array}$ & Нет \\
\hline $\begin{array}{l}\text { Хроническая } \\
\text { обструктивная болезнь } \\
\text { легких }\end{array}$ & + & - & + & + \\
\hline $\begin{array}{l}\text { Артериальная } \\
\text { гипертония }\end{array}$ & + & + & + & + \\
\hline Курение & + & - & - & + \\
\hline Клапанные пороки & & Нет & & Нет \\
\hline $\begin{array}{l}\text { Хроническая болезнь } \\
\text { почек }\end{array}$ & - & $\begin{array}{l}\text { Скорость клубочковой } \\
\text { фильтрации } 62\end{array}$ & - & - \\
\hline Ожирение & Индекс массы тела 27 & Индекс массы тела 22 & Индекс массы тела 25 & Индекс массы тела 23 \\
\hline $\begin{array}{l}\text { Инфаркт миокарда } \\
\text { в анамнезе }\end{array}$ & - & - & + & - \\
\hline
\end{tabular}

Операция проводилась без использования рентгеновского оборудования. Радиочастотная энергия подавалась точка за точкой, по окружности вокруг ЛВ приблизительно в 1 см устья легочных вен. Использовалась система трехмерной навигации Carto $3^{\text {тм }}$ biosense webster. Использовался абляционный электрод с возможностью измерения силы прижатия - ThermoCool $^{\circledR}$ SmartTouch ${ }^{\circledast}$ производство biosense webster. Далее проводилась антральная изоляция легочных вен. Использовался диагностический двадцатиполюсной электрод Lasso производство biosense webster. легочных вен оценивалась по следующим параметрам: уменьшение амплитуды сигнала с диагностического электрода более чем в 5 раз, отсутствие проведение импульса (блок входа и блок выхода). После удаления инструментов из левого предсердия мы измеряли время правопредсердного проведения по стандартной методике [13].

Пациентам из второй группы проводилась КТП перешейка с помощью абляционного катетера с охлаждаемым наконечником 3,5 мм. Процедура осуществлялась по стандартной методике. Критерии эффективности блока в КТП наличие двунаправленного блока проведения.

Период наблюдения составил 2 года. Последующее наблюдение за пациентами проводилось через 24 ч после процедуры с ЭКГ-мониторингом. Антикоагулянтная терапия возобновлялась через 6 часов после операции. Bсе пациенты продолжали антикоагулянтное лечение не менее 3 месяцев. Пациентам с рецидивирующей ФП в течение 3 месяцев после абляции была проведена кардиоверсия, и по показаниям был назначен антиаритмический препарат. Все пациенты проходили клиническую оценку у одного и того же врача через 3, 6, 12, 24 месяца, включая ЭКГ по 12 отведениям и 2-дневный ХМ-ЭКГ. Через 3 месяца наблюдения мы отменяли антиаритмическую терапию, если у пациентов не было ФП и ТП на ХМЭКГ. Пациентам проводилась повторная абляция если после трех месяцев сохранялись симптомные пароксизмы ФП или ТП.

\section{Результаты}

У всех пациентов интраоперационно удалось получить критерии изоляции устьев легочных вен. Двухсторонний истмус блок удалось получить у всех пациентов. В течение слепого периода наблюдения (3 месяца) значимых отличий в частоте возникновения пароксизмов ФП между двумя группами не выявлено ( $\varphi^{*} э м п=0.787$, р>0.05): частота пароксизмов составила 23,81\% и $19,05 \%$ в первой и второй группах соответственно. Устойчивые пароксизмы ФП не регистрировались у 76,19\% и 71,43\% в первой и второй группах соответственно ( $\varphi^{*} э$ мп = 0.353, p>0.05). Случаев ТП во второй группе не наблюдалось. В первой группе наблюдалось 3 (14,29\%) случая ТП в течение одного года наблюдения. Далее представлена таблица № 4 по факторам риска рецидивных пациентов (ТП) из первой группы. 
В течение двух лет устойчивые пароксизмы ФП не регистрировались у 66,67\% и 61,9\% в первой и второй группах соответственно ( $\varphi^{*} э$ мп $\left.=0.324, p>0.05\right)$. Случаев ТП во второй группе через 2 года наблюдения не было. В первой группе в течение двух лет было определено 4 случая ТП $(19,05 \%) \mathrm{X}^{2}=17.0, \mathrm{p}<0.05$.

Среднее время операции в первой группе составило $86 \pm 12$ минут, средне время операции во второй группе составило 97 \pm 11 минут $\mathrm{t}_{\text {эмn }}=3,2, \mathrm{t}_{\kappa p}=2,02, \mathrm{p}<0,05$. Время флюороскопии в обеих группах 0.

Необходимо дополнить, что 4 случая рецидивов ТП В первой группе потребовали повторной госпитализации.

Осложнения в обеих группах представлены гематомами в области пункции бедренной вены. В первой группе 1 пациент (4,76\%). Во второй группе 1 пациент (4,76\%), p>0,05 ДИ=0,95\%.

Всем пациентам с рецидивом ФП была повторно проведена изоляция устьев легочных вен. Пациентам с выявленным ТП была проведена абляция КТП и проверка изоляции устьев легочных вен.

Проблема превентивного блока в КТП ранее изучалась различными авторами. Далее представлены результаты проведенных ранее исследований.

Группа авторов под руководством J Pontoppidan и др. в 2009 году предоставили отдаленные результаты интервенционного лечения у 149 пациентов, которые были рандомизированны на две группы. Изоляция легочных вен проводилась в первой группе (76 человек), изоляция легочных вен и абляция КТП во второй группе (73 человека). Срок наблюдения составил 12 месяцев. Спустя 12 месяцев авторы не получили статистически значимой разницы между группами пациентов: у 84\% из первой группы и 85\% из второй группы сохранялся синусовый ритм, разницы в возникновении ТП получено не было. Повторный холтеровский мониторинг (ХМ-ЭКГ) продемонстрировал $67 \%$ эффективность в течение 1 года наблюдения. Включая повторные процедуры, клинический эффект был отмечен в 64\% случаев. Таким образом, проведение одномоментной процедуры у пациентов без документированного ТП по мнению авторов не показано.

В 2018 году группа авторов провели исследование [7]. Количество пациентов 882. Пациенты были рандомизированны на две группы. В первой группе проводили только изоляцию легочных вен $\mathrm{n}=441$. Во второй группе проводили изоляцию легочных вен одномоментно с аблацией КТП $\mathrm{n}=441$. Период отдаленного наблюдения составил 2 года. Результаты: пароксизмы ФП и ТП отсут- ствовали у 79\% пациентов из первой группы и у 79\% из второй. Авторы сформулировали вывод: превентивная абляция КТП не приводит к снижению частоты возникновения ФП и ТП в постоперационном периоде.

Однако, в этих исследованиях, авторы не учитывали факторы риска возникновения ТП.

Данные факторы были изучены в исследовании [8]. Период наблюдения $33.0 \pm 12.2$ лет. Количество пациентов 5209, у 112 (2.1\%) пациентов, вошедших в исследование, было диагностировано ТП. В многофакторном анализе курение (ОШ) 2,84; 95\% [ДИ], 1,54-5,23; P <0,001), удлиненный интервал PR (1,28, на стандартное отклонение (32 мс); 95\% ДИ от 1,03 до 1,60; $\mathrm{P}=0,03)$, инфаркт миокарда в анамнезе (ОШ 2,25; $95 \%$ ДИ от 1,05 до 4,80; $\mathrm{P}=0,04)$ и наличие хронической сердечной недостаточности (ХСН) (ОШ) 5,22; 95\% ДИ, от 1,26 до 21,64; P = 0,02) оставались статистически значимо связанными с повышенным риском возникновения ТП. К преимуществам данного исследования можно отнести большую выборку пациентов, тщательное определение клинических факторов риска и длительный период наблюдения.

Также данные факторы риска были подтверждены в исследованиях [10-12].

Другой фактор риска, а именно время правопредсердного проведения, изучался в исследовании [13]. В работе отмечено, что ТП является распространенным исходом после изоляции легочных вен. В исследовании авторы измеряли время правопредсердного проведения до и после изоляции легочных вен, а также изучалась их связь с развитием ТП. По данным авторов ТП связано с пролонгированием времени проведения в правом предсердии. Абляция во время изоляции правых легочных вен может спровоцировать замедление проводимости, которое может привести к возникновению ТП в отдаленные сроки после операции.

Также данные факторы риска мы изучали в работе: «Анализ факторов риска возникновения типичного трепетания предсердий у пациентов, которым проводится изоляция устьев легочных вен». Результаты нашего исследования совпадают с ранее опубликованными результатами о факторах риска возникновения ТП: ХОБЛ (ОШ 25,4; ДИ 95\%; 10,991-58,609), ХСН (ОШ 7,434; ДИ 95\%; 3,209-17,225), удлинённый интервал p-r (64.29\%) (ОШ 5,77; дИ 95\%; 2,288-14,5), инфаркт миокарда в анамнезе (ОШ 6,591; ДИ 95\%; 2,447-17,751), курение (ОШ 11,034; ДИ 95\%; 4,849-25,112).

В текущую работу были включены пациенты с факторами риска возникновения ТП, что и отличает нашу работы от ранее проведенных исследований. Результаты 
нашего исследования совпадают с ранее опубликованными результатами о факторах риска возникновения ТП. У пациентов, у которых ТП возникло в постоперационном периоде, присутствуют несколько факторов риска ТП, что возможно, свидетельствует о потенцировании факторов риска в отношении возникновения ТП.

Возможно проведение превентивной абляции пациентам с факторами риска ТП, особенно пациентам с наличием нескольких факторов риска ТП. По нашим данным превентивная аблация КТП пациентам с факторами риска возникновения ТП значимо снижает частоту возникновения ТП в отдаленном периоде наблюдения. Превентивная аблация КТП увеличивает время процедуры и не увеличивает число интраоперационных осложнений. По нашим данным превентивная абляция КТП не влияет на возникновение осложнений и способна снизить число последующих госпитализаций и повторных операций.

\section{Список сокращений}

ДИ-доверительный интервал, КТП-каватрикуспидальный перешеек, ЛКА-левая коронарная артерия, МПП-межпредсердная перегородка, НПВ-нижняя полая вена, ОШ-отношение шансов, ПКА-правая коронарная артерия, ТП-трепетание предсердий, ФВ-фракция выброса, ХМ-ЭКГ-суточное мониторирование по Холтеру, ФП-фибрилляция предсердий, ХОБЛ-хроническая обструктивная болезнь легких, ХСН-хроническая сердечная недостаточность, ЭКГ-электрокадиография.

\section{ЛИТЕРАТУРА}

1. А.В. Струтынский. Электрокардиограмма. Анализ и интерпретация.- М: МЕДпресс-информ, 2009.- C. 120-123.— 224 с [A.V. Strutynskij. Elektrokardiogramma. Analiz i interpretaciya. — M: MEDpress-inform, 2009. — S. 120-123. — 224 s (in Russ)]

2. Mortality, morbidity, and quality of life after circumferential pulmonary vein ablation for atrial fibrillation: outcomes from a controlled nonrandomized long-term study. Pappone C, Rosanio S, Augello G, Gallus G, Vicedomini G, Mazzone P, Gulletta S, Gugliotta F, Pappone A, Santinelli V, Tortoriello V, Sala S, Zangrillo A, Crescenzi G, Benussi S, Alfieri 0J Am Coll Cardiol. 2003 Jul 16; 42(2):185-97.

3. Atrial fibrillation originating from persistent left superior vena cava. Hsu LF, Jaïs $P$, Keane D, Wharton JM, Deisenhofer I, Hocini M, Shah DC, Sanders $P$, Scavée $C$, Weerasooriya R, Clémenty J, Haïssaguerre M Circulation. 2004 Feb 24; 109(7):828-32.

4. Wyndham CRC. Atrial Fibrillation: The Most Common Arrhythmia (неопр.) // Texas Heart Institute Journal.— 2000.— T. 27, № 3.— C. $257-267$.

5. Long-term outcomes after catheter ablation of cavo-tricuspid isthmus dependent atrial flutter: a meta-analysis.Pérez FJ, Schubert CM, Parvez B, Pathak V, Ellenbogen KA, Wood MA Circ Arrhythm Electrophysiol. 2009 Aug; 2(4):393-401.

6. 2019 AHA/ACC/HRS Focused Update of the 2014 AHA/ACC/HRS Guideline for the Management of Patients With Atrial Fibrillation: A Report of the American College of Cardiology/American Heart Association Task Force on Clinical Practice Guidelines and the Heart Rhythm Society in Collaboration With the Society of Thoracic Surgeons

7. Int J Cardiol. 2018 May 15;259:82-87. doi: 10.1016/j.jicard.2018.01.025. Impact of prophylactic cavotricuspid isthmus ablation in atrial fibrillation recurrence after a first pulmonary vein isolation procedure. Mesquita J, Ferreira AM, Cavaco D, Carmo P, Madeira M, Freitas P, Costa FM, Morgado F, Mendes M, Adragão P.

8. Atrial flutter: Clinical risk factors and adverse outcomes in the Framingham Heart Study Heart Rhythm $2016 \mathrm{Jan} ; 13$ (1):233-40. Epub 2015 Jul 28. Faisal Rahman, Na Wang, Xiaoyan Yin, Patrick T Ellinor, Steven A Lubitz, Paul A LeLorier, David D McManus, Lisa M Sullivan, Sudha Seshadri, Ramachandran S Vasan, Emelia J Benjamin, Jared W Magnani. doi: 10.1016/j.hrthm.2015.07.031

9. Europace. 2013 Dec;15(12):1777-83. Epub 2013 Jun 19. Prevalence and electrophysiological characteristics of typical atrial flutter in patients with atrial fibrillation and chronic obstructive pulmonary disease.Hayashi T, Fukamizu S, Hojo R, Komiyama K, Tanabe Y, Tejima T, Nishizaki M, Hiraoka M, Ako J, Momomura S, Sakurada H. doi: 10.1093/europace/eut158

10. Garcia-Cosio F, Pastor Fuentes A, Nunez Angulo A. Arrhythmias (IV). Clinical approach to atrial tachycardia and atrial flutter from an understanding of the mechanisms. Electrophysiology based on anatomy. Rev Esp Cardiol (Engl Ed) 2012;65:363-375.]

11. Pacing Clin Electrophysiol. 2008 Apr;31(4):432-7.2008.01012.x. Anatomic and electrophysiologic differences between chronic and paroxysmal atrial flutter: intracardiac echocardiographic analysis. Ohkubo K, Watanabe I, Okumura Y, Ashino S, Kofune M, Kawauchi K, Yamada T, Kofune T, Hashimoto K, Shindo A, Sugimura H, Nakai T, Kunimoto S, Saito S, Hirayama A. doi: 10.1111/j.1540-8159

12. J Am Coll Cardiol. 1990 Dec;16(7):1639-48.Demonstration of an area of slow conduction in human atrial flutter. Olshansky B, Okumura K, Hess PG, Waldo AL.J Am Coll Cardiol. 2000 Dec; 36(7):2242-6. Incidence and predictors of atrial flutter in the general population. Granada J, Uribe W, Chyou PH, Maassen K, Vierkant R, Smith PN, Hayes J, Eaker E, Vidaillet $H$.

13. Europace. 2011 Jul;13(7):942-8. Epub 2011 Mar 21.Changes in low right atrial conduction times during pulmonary vein isolation for atrial fibrillation: correlation with inducibility of typical right atrial flutter. Dizon J, Biviano A, Whang W, Ehlert F, Garan H. doi: 10.1093/europace/eur033 